Mater. Res. Soc. Symp. Proc. Vol. 1384 @ 2012 Materials Research Society

DOI: 10.1557/opl.2012.363

\title{
Synthesis of Mesoporous Gadolinium Doped Ceria - Platinum Composite
}

Hoi Yung, Kwong-Yu Chan and Frank Leung-Yuk Lam

Department of Chemistry, The University of Hong Kong, Pokfulam Road, Hong Kong

\begin{abstract}
Oxygen reduction in SOFC cathodes has long been the rate determining step in SOFC operations, mixed ionic-electronic conductors (MIECs) and/or forming composite between cathode and electrolyte materials have been common strategies in order to aid the cathode kinetics. We demonstrate here a viable synthesis route to impregnate mesopores with high loading of platinum towards a mesoscale bicontinuous material that composed of channels of a fast ionic conductor, i.e. gadolinium doped ceria (GDC) intertwined with channels of a good electronic conductor, i.e. Pt. This highly structural composite material holds the promise of a high performing cathode in SOFC.
\end{abstract}

\section{INTRODUCTION}

Solid oxide fuel cell (SOFC) is a very efficient electrochemical device. Its commercialization is crucial to a more sustainable energy consumption pattern based on renewable energies. Owing to its high operating temperatures $\left(800-1000^{\circ} \mathrm{C}\right)$, expensive catalysts such platinum can be replaced. An additional feature of SOFC is the possibility to operate as an electrolyzer which splits water into its constituents with equally high efficiency ${ }^{1}$. Therefore, SOFC can also be used as an efficient energy converter in energy storage where off peak power can be stored in the form of a chemical fuel.

As operating temperature decreases to the intermediate range $\left(600-800^{\circ} \mathrm{C}\right)$, electrode kinetics slows down significantly since all SOFC processes are thermally activated. Reduced ionic conductivity in solid electrolytes are usually compensated by making the electrolyte layer thinner The electrode kinetics is improved by using a mixed ionic-electronic conductor (MIEC) and further improved by addition of an ionic conductor such as the electrolyte material itself to aid oxygen ion diffusion ${ }^{2,3}$. These strategies all aims at increasing the length of three phase boundaries (tpb) where key oxygen reduction steps take place. Classic examples are $\mathrm{La}_{0.6} \mathrm{Sr}_{0.4} \mathrm{Co}_{0.2} \mathrm{Fe}_{0.8} \mathrm{O}_{3-\delta}(\mathrm{LSCF})$ and Gd doped ceria (GDC) composite studied by Dusatres, et al ${ }^{2}$. These composite cathodes are usually made by randomly mixing the individual phases in powder form followed by partial sintering forming a porous structure that adhere to the electrolyte. The random nature of mixing is often modeled by the effective medium percolation theory (EMPT) which predicts a sharp transition of performance at a certain ratio of the individual phases. In reality, since particle sizes are not uniform and particle shape is irregular, threshold ratio is hard to be obtained. Besides, the improvement in performance is marginal partly due to the fact that each phase is not fully interconnected so that reduced oxygen ions cannot be carried away efficiently. Efforts have been made to further optimize the cathode structure, for instance, functionally graded electrodes ${ }^{4}$ or impregnation of one phase into a porous network of another phase ${ }^{5}$. Jiang et al. ${ }^{6}$ was the first one to attempt the strategy using incipient wetness impregnation on SOFC electrodes. The results were positive with significant increase in cathode performance. 
In the last decade or so, a new strategy so called "hard templating" became possible, mesoporous silica networks can be synthesized using amphiphilic surfactants ${ }^{7,8}$ The silica network was then used as templates to synthesize highly crystalline ordered mesoporous materials with uniform pore size in the nanometer range and wall thickness of the same order ${ }^{9-}$

${ }^{16}$. These mesoporous metal oxides are strong enough to be self-supportive and they usually have surface area comparable to nano-particles of similar size. One common feature of these templated metal oxides is that they are often single-crystalline-like as a result of confinement effect $^{10}$.

We propose here the idea of Pt impregnation into mesopores using a organometallic precursor and as loading increases, forming a bi-continuous composite electrode material consist of two intertwining networks of materials one being mainly electronic conductor and the other being mainly ionic conductor. Each network has a wall thickness less than $10 \mathrm{~nm}$ to maximize the length of the triple phase boundary.

\section{EXPERIMENTAL}

The method used for making KIT-6 silica is recorded elsewhere ${ }^{8}$. Hydrothermal temperature of $60^{\circ} \mathrm{C}$ was chosen for this study. Total pore volume of the KIT-6 template was determined by BET (Micrometritis, ASAP2020) to be $0.68 \mathrm{~cm}^{3} \mathrm{~g}^{-1}$. KIT-6 template follows a gyroid structure which consists of two interpenetrated network of channels of the same shape and size but shifted in space by half a unit cell. The pore dimensions where determined by BET to have a modal diameter of $5.2 \mathrm{~nm}$.

Stoichiometric amount of cerium nitrate hexahydrate $\left(\mathrm{Ce}\left(\mathrm{NO}_{3}\right)_{3} \cdot 6 \mathrm{H}_{2} \mathrm{O}\right)$ and gadolinium nitrate hexahydrate $\left(\mathrm{Gd}\left(\mathrm{NO}_{3}\right)_{3} \cdot 6 \mathrm{H}_{2} \mathrm{O}\right)$ with a target composition of $\mathrm{Ce}_{0.8} \mathrm{Gd}_{0.2} \mathrm{O}_{2-\delta}$ was dissolved in $18.2 \mathrm{M} \Omega$ deionized water forming a saturated solution. Since water of crystallization varies, the actual amount was determined by TGA and adjusted accordingly.

For template GDC, the bi-solvent method was used ${ }^{11}$. For a typical synthesis, $6 \mathrm{~g}$ of KIT6 silica powder was dispersed in octane. The suspension was stirred for 3 hours before $4.08 \mathrm{ml}$ of saturated precursor solution was added according to the above measured pore volume. Once all the precursor solution entered the mesopores, the template particles sank to the bottom. The mixture was retrieved and dried in an air oven at $60^{\circ} \mathrm{C}$ for 1 hour. The dried powder was calcined in a vented top-hat furnace at $550^{\circ} \mathrm{C}$ for 6 hours to decompose the precursor into oxide. The template was removed by multiple washes with large amount of hot $2 \mathrm{M} \mathrm{NaOH}$ solution followed by 10 washes using deionized water and ethanol and drying in oven overnight. The ordered mesoporous GDC (OMG) had a pore volume of $0.245 \mathrm{~cm}^{3} \mathrm{~g}^{-1}$.

The OMG was mixed in 1:1 weight ratio with Dimethyl(1,5-cyclooctadiene)platinum(II), 99\% purity, from Strem Chemicals INC. (DMCP) in an agate mortar. The mixture was then transferred to an alumina crucible boat placed in a tube furnace. The heating ramped from room temperature to $240^{\circ} \mathrm{C}$ at $5^{\circ} \mathrm{C} / \mathrm{min}$ in a flow of ammonia at $200 \mathrm{ml} / \mathrm{min}$ and held for 2 hours before the gas was switched to hydrogen and the temperature rose to $390^{\circ} \mathrm{C}$ at the same ramp rate. After dwelling for 2 hours, the setup was allowed to cool down in hydrogen. The process was repeated in order to increase the platinum loading. Weights and wide-angle XRD patterns were used to estimate the loading. Another sample with precursor placing separately upstream from OMG was also prepared to show the effect of vapor phase impregnation alone. 
Wide-angle XRD was performed with Bruker D8, HRTEM was taken with Philips Tecnai G2 20 S-TWIN and the FEG-HRTEM was performed using JEOL 2010F. Since both materials are polycrystalline with similar cubic lattice structures, cross-sections were prepared by mounting with epoxy between two pieces of Si wafer followed by polishing and low angle ion beam milling using Fischione Model 1010.

\section{RESULTS AND DISCUSSION}

\section{Synthesis of mesoporous GDC}

In this study, ionic conducting network made of GDC was first synthesized by hardtemplating and then impregnated the structure with an electronic conducting phase, i.e. platinum using metal-organic chemical vapor deposition (MOCVD).

After a maximum of $5 \mathrm{Pt}$ impregnations, significant loading $\sim 33 \mathrm{Vol} \%$ of total internal pore volume determined from weight, was archived. Fig. 1a shows the wide angle XRD pattern of templated GDC with good match to $\mathrm{Ce}_{0.8} \mathrm{Gd}_{0.2} \mathrm{O}_{2-\mathrm{x}}$ (PDF\# 50-0201) with a lattice parameter of $5.42 \AA$. An interesting bimodal distribution of pore sizes $(5.1$ and $11.5 \mathrm{~nm})$ was observed in both TEM (fig. 1b) and BET pore size distribution (fig. 1c). Similar observations were reported by Kim et al. ${ }^{17}$ for carbon and Jiao et al. ${ }^{14}$ for nickel oxide. Jiao et al. concluded that the bimodal distribution was caused by the lack of micropores between the two intertwined channels. When precursor could access two adjacent channels, i.e. via the micropores, the templated GDC would have a wall thickness equivalent to the pore size of and a pore size of the wall thickness of the KIT-6 template. Whereas when precursor could not access adjacent channels, one of the channels would be filled; the templated structure would still have a wall thickness equivalent to the pore size of the template, but the pore size would be equivalent to one channel plus two wall thicknesses of the template.

a)

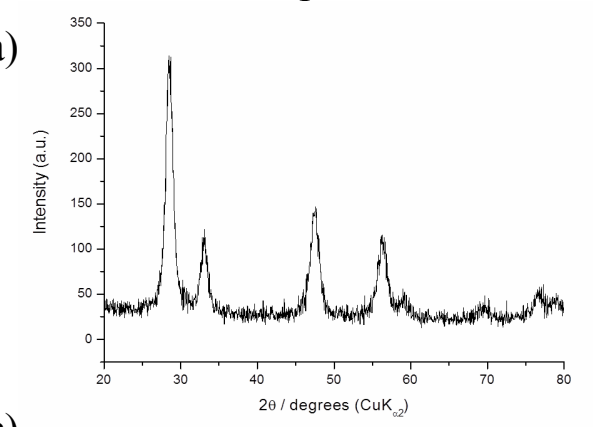

c)

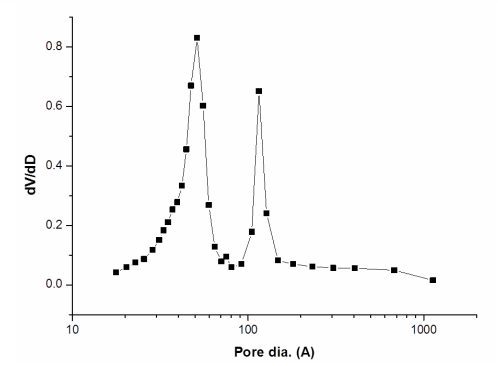

b)

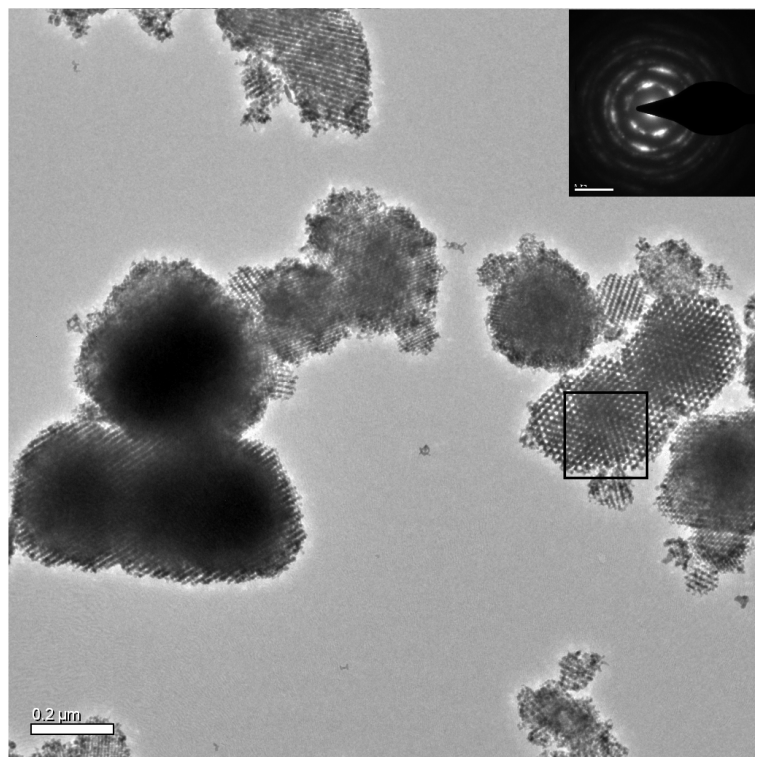

Fig. 1 a) Wide-angle XRD, b) HRTEM image with SAED, and c) pore distribution obtained from BET measurement of the GDC replica after silica removal. 


\section{Implications of silica contaminations}

Silica contamination is the most studied and the greatest cause of ionic conductivity drops in solid electrolyte such as GDC. The presence of $\mathrm{SiO}_{2}$ phase could affect ionic conduction in two ways ${ }^{18}$. Small quantity would dissolve into the GDC crystal lattice at high temperatures. The strong Si-O bond would hinder the formation of oxygen vacancies, hence reduce ionic conductivity. The majority amount of $\mathrm{SiO}_{2}$ would remain at grain boundaries, depending on the Gd dopant level, $\mathrm{Gd}_{2} \mathrm{SiO}_{5}, \mathrm{Gd}_{9.33}\left(\mathrm{SiO}_{4}\right)_{6} \mathrm{O}_{2}$ and $\mathrm{Gd}_{2} \mathrm{SiO}_{7}$ could be formed between $\mathrm{Gd}_{2} \mathrm{O}_{3}$ and $\mathrm{SiO}_{2}$. This scavenging effect of Gd helps when $\mathrm{Si}$ is in the ppm level, however, we found that silica could not be fully removed by hot $\mathrm{NaOH}$ even after 10 washes. Significant amount $\sim 10$ at $\%$ could be present. The difficulty of silica removal was reported in many occasions and therefore related to the technique itself. However, it is worth noting there were several key differences between the synthesis route used in this study and a typical powder-based preparation route. First of all, solid electrolyte was sintered with intentional addition of silica; silica would certainly reside at GBs perpendicular to the ionic current flow; whereas in our study, because of the unique geometry of a reverse gyroid, silica should only be located on the pore surface. Besides, temperature used to sinter a solid electrolyte is usually above $1200^{\circ} \mathrm{C}$, significantly higher than the $550^{\circ} \mathrm{C}$ used in this study. Therefore, it is unlikely for Si to enter GDC crystal lattice and reside in GBs in the pore walls perpendicular to the ion flow. The main issues are the oxygen diffusion across GDC-air interface.

\section{Platinum impregnation}

A common composite cathode material is Ag mixing with the electrolyte material. Silver, despite a metal, possess oxygen ion conductivity ${ }^{19}$ and the highest electronic conductivity. It would be a very good candidate for the composite structure we proposed. However, low melting point and photo sensitivity of its metal-organic precursors makes it a challenging material to work with. Pt was chosen because of the precursor could be handled with ease.

The Pt precursor chosen (DMCP) melts at $103 \sim 105^{\circ} \mathrm{C}$ and at the same time begin to vaporize. The cyclooctadiene ligand comes off at relative low temperatures, and the lone pair of the remaining molecules attached to the GDC surface. The molten precursor was also expected to fill the pore channels by capillary force, providing a second route of impregnation when decomposed. It can be seen from Fig. $2 \mathrm{a}$ and $2 \mathrm{~b}$ that Pt loading increased with increasing number of impregnations. This is confirmed by EDX (fig. 2d and 2e) during FEG-HRTEM and supported by the increasing Pt peaks in the XRD patterns (fig. 2c). The darker region in fig. $2 b$ and $2 \mathrm{c}$ gave a much stronger Pt peak compared to the lighter region is a strong indication of their compositions. Weight of the sample was monitored between each impregnation that gives an estimate of $38 \%$ fill

An interesting fact was that most of the Pt recited inside the pores. Only one layer of $\mathrm{Pt}$ nano-particles was observed on the outer surface in top right corner of fig.2b. Fig. $2 \mathrm{~g}$ shows a sample of mesoporous GDC impregnated 8 times by vapor phase only. Significantly less Pt was observed which implies that liquid phase impregnation played an important role. Vapor phase deposition alone is slow, but it could be automated using a modern CVD system. 

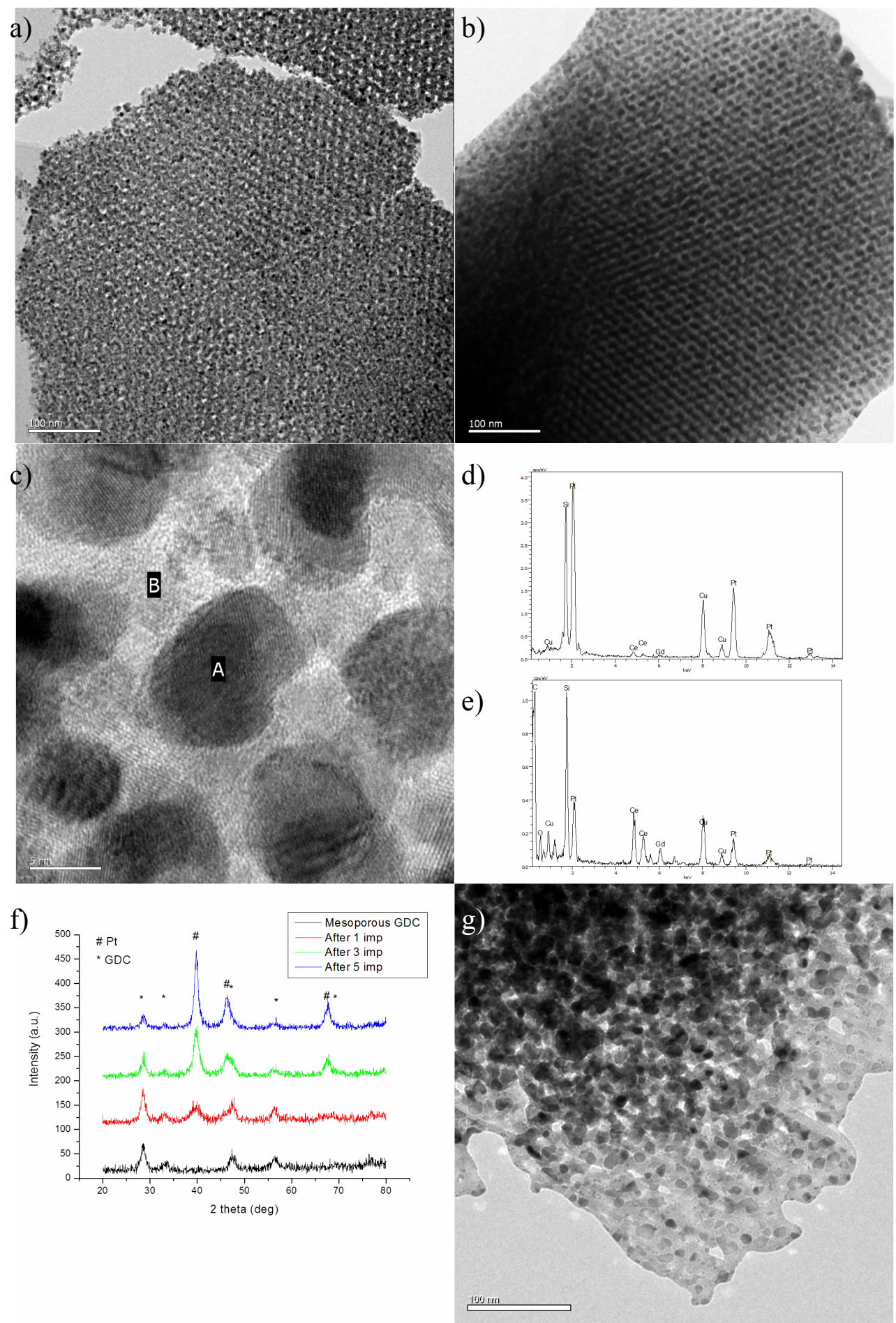

Fig. 2 FEG-HRTEM images showing the cross-section of mesoporous GDC after a) 1 and b) 5 impregnations with higher magnification of b) shown in c), EDX performed on selected region of A and B shown in d) and e). f) XRD of the sample powder after 1, 3 and 5 impregnations showing increase in Pt peaks; and HRTEM image showing sample cross-section after $8 \mathrm{Pt}$ impregnations by vapor-phase deposition alone $\mathrm{g}$ ).

\section{CONCLUSIONS}


Mesoporous GDC was synthesized by silica hard-templating, followed by $\mathrm{Pt}$ impregnation in attempt to form a bicontinuous composite as SOFC cathode material. Further work is required such as increasing Pt loading by using a automated CVD system and local electrical measurement to prove Pt phase connectivity.

\section{ACKNOWLEDGMENTS}

The authors would like to thank Mr. Y.F. Chan and Mr. T.K. Cheung for their advices

and assistance in electron microscopy and the support of University Development Fund given to the Initiative on Clean Energy and Environment (ICEE).

\section{REFERENCES}

1. M. A. Laguna-Bercero, S. J. Skinner and J. A. Kilner, Journal of Power Sources 192 (1), 126-131 (2009).

2. V. Dusastre and J. A. Kilner, Solid State Ionics 126 (1-2), 163-174 (1999).

3. Y. J. Leng, S. H. Chan, K. A. Khor and S. P. Jiang, Journal of Applied Electrochemistry 34 (4), 409-415 (2004).

4. $\quad$ N. T. Hart, Journal of Materials Science 36 (5), 1077-1085 (2001).

5. S. P. Jiang, Y. J. Leng, S. H. Chan and K. A. Khor, Electrochemical and Solid-State Letters 6 (4), A67-A70 (2003).

6. S. P. Jiang, Y. Y. Duan and J. G. Love, Journal of The Electrochemical Society 149 (9), A1175-A1183 (2002).

7. P. Y. Dongyuan Zhao, Nick Melosh, Jianglin Feng, Bradley F. Chmelka, Galen D. Stucky, Advanced Materials 10 (16), 1380-1385 (1998).

8. T.-W. Kim, F. Kleitz, B. Paul and R. Ryoo, Journal of the American Chemical Society 127 (20), 7601-7610 (2005).

9. W. Yue and W. Zhou, Progress in Natural Science 18 (11), 1329-1338 (2008).

10. C. Dickinson, W. Zhou, R. P. Hodgkins, Y. Shi, D. Zhao and H. He, Chemistry of materials 18 (13), 3088-3095 (2006).

11. K. Jiao, B. Zhang, B. Yue, Y. Ren, S. Liu, S. Yan, C. Dickinson, W. Zhou and H. He, Chemical Communications (45), 5618-5620 (2005).

12. Y. Wang, X. Yuan, X. Liu, J. Ren, W. Tong, Y. Wang and G. Lu, Solid State Sciences 10 (9), 1117-1123 (2008).

13. M. Mamak, N. Coombs and G. Ozin, Advanced materials 12 (3), 198-202 (2000).

14. F. Jiao, A. H. Hill, A. Harrison, A. Berko, A. V. Chadwick and P. G. Bruce, Journal of the American Chemical Society 130 (15), 5262-5266 (2008).

15. Yue and Zhou, Chemistry of materials 19 (9), 2359-2363 (2007).

16. E. Rossinyol, E. Pellicer, A. Prim, S. Estradé, J. Arbiol, F. Peiró, A. Cornet and J. Morante, Journal of Nanoparticle Research 10 (2), 369-375 (2008).

17. T.-W. Kim and L. A. Solovyov, Journal of Materials Chemistry 16 (15), 1445-1455 (2006).

18. T. S. Zhang, J. Ma, S. H. Chan, P. Hing and J. A. Kilner, Solid State Sciences 6 (6), $565-$ 572 (2004).

19. I. Kontoulis and B. C. H. Steele, Solid State Ionics 47 (3-4), 317-324 (1991). 